\title{
Health Systems Research in leprosy control-what contributions can it make?
}

\author{
P. LEVER, L. BIJLMAKERS, P. ZWANIKKEN \& \\ P. SAUNDERSON* \\ Royal Tropical Institute, Amsterdam, the Netherlands; \\ *Director Leprosy and Tuberculosis Control Programme ALERT, \\ Ethiopia
}

Accepted for publication 22 April 1998

\begin{abstract}
Summary The paper describes a Health Systems Research (HSR) training programme which took place at the All Africa Leprosy, Tuberculosis and Rehabilitation Training Centre (ALERT) in Ethiopia. The training consisted of three stages: an initial workshop focussing on protocol development, followed by a fieldwork period and a data analysis and report writing workshop. Twenty participants, divided over four groups, took part in the training and carried out the research alongside their dayto-day professional commitments. Three of the projects were concerned with prevention of disabilities, one with integration of the leprosy programme into the general health services. Based on the findings of their research, each group produced a set of recommendations and a plan of action for the implementation of these recommendations. The contribution of HSR to leprosy control is discussed.
\end{abstract}

\section{Introduction}

Health Systems Research (HSR) is a type of research that is concerned with improving people's health by enhancing the efficiency and effectiveness of the health system as an integral part of the overall process of socio-economic development.

More specifically, HSR may be used to help solve practical problems that are encountered by health programme managers, health staff and/or community members. Problems that can be addressed through HSR may be related to the delivery of specific health services; to proper use or targeting of resources, such as human resources, physical structures, materials and finance; and to the relative merits and demerits of certain health policies or programmes.

The main characteristics of HSR are:

- it is problem based and has a strong orientation towards seeking feasible, practical and affordable solutions to these problems;

Correspondence to: Dr P. Lever, Leprosy and Tuberculosis Unit Royal Tropical Institute (KIT), Wibautstraat 137 j 1097 DN, Amsterdam, The Netherlands 
- it is participatory: there is a close dialogue between those who identify the problems to be studied and who are the main potential users of the results-the health programme managers and policy makers - and those who search for the facts and suggest alternative solutions - the researchers; in many cases, the programme managers are the researchers themselves;

- it is multidisciplinary: contributions are obtained from a variety of disciplines, which may include health science, epidemiology, sociology, anthropology, economics, public administration and statistics, through a team work approach.

Because of the variation in types of problems that qualify for HSR studies, there is no single HSR methodology. Each problem requires its own methodology to quantify the problem under study, identify its various direct and indirect causes, measure the relative importance of each cause and explore possible solutions. Efforts have been made, however, to give structure to the process of developing HSR methodologies. One of the best resources that are currently available is the manual Designing and Conducting Health Systems Research Projects, ${ }^{1}$ which is published by WHO and IDRC. The manual consists of two parts (the first about proposal development and fieldwork and the second about data analysis and report writing) and is the second volume of a five-volume series, issued by WHO/IDRC under the title Health Systems Research Training Series.

In this paper, we describe an HSR module based on this manual, whereby four research projects related to leprosy were developed, implemented and subsequently analysed at the All Africa Leprosy, Tuberculosis and Rehabilitation Training Centre (ALERT) in Addis Ababa, Ethiopia. Possible implications for use in other leprosy control projects will be discussed.

\section{Health Systems Research at the All Africa Leprosy, Tuberculosis and Rehabilitation Training Centre (ALERT)}

ALERT was set up over 30 years ago as a leprosy training centre for Africa. The main focus is leprosy and rehabilitation, although in recent years tuberculosis has become an important part of ALERT's activities in both training and service provision. The organization comprises three divisions:

- The Training Division, which organizes and manages all the formal training activities undertaken by ALERT.

- The Hospital Services Division, which runs a 235-bed hospital with medical, surgical and ophthalmology departments.

- The Leprosy/TB Control Division, which manages a control programme in central Ethiopia, an area with a total population of 13 million and a leprosy prevalence of $1 \cdot 1$ per 10,000 .

Prevention of disability (POD) activities have become an increasingly important part of leprosy work as the prevalence of registered cases has decreased. One of the major concerns at ALERT is the fact that the rate of disability in both new and treated cases has remained at a constant and unacceptably high level, despite all the resources that have been available to the organization, and the original research that has been conducted over the years. Each division of ALERT therefore sees POD as a central issue, with many problems still to be resolved.

For the Training Division, a major challenge is to develop training guidelines for POD in 
the now typical situation in which leprosy work is integrated into the general health services. For the Control Division, there is a need to find out why the disability rate in new cases remains high and then design specific and targeted programmes of health education to reverse the situation. For the Hospital Division, better ways of preventing further disability are needed, whether in the management of acute neuritis or better self care in patients with permanent nerve damage. There is clearly a lot of overlap in the activities of the three divisions, and many of the issues in POD are looked at jointly.

Technical expertise and financial resources were generally not lacking at ALERT, and therefore it became clear that operational aspects of the work would have to be examined in detail if POD activities and outcomes were to be improved. This would involve management issues such as quality, efficiency and motivation on the one hand, and, perhaps more importantly, a greater emphasis on addressing and adapting to the patient's own socio-cultural situation and beliefs, on the other. These operational problems can most appropriately be investigated using the HSR methodology, and this was suggested by the Medical Advisory Committee of ALERT.

A typical HSR training course as proposed in the manual 'Designing and Conducting $H S R$ Projects' described above, consists of three stages: two workshops and a fieldwork period.

The first workshop, aimed at the development of research proposals, took place during the first 2 weeks of June 1996. Of the 20 participants, the large majority were ALERT staff, coming from all three divisions: Control, Training and the Hospital. Sixteen of the participants were medical doctors (public health physicians, surgeons, dermatologists, and a specialist in internal medicine), two were physiotherapists, one a nurse and one a social worker. There were three facilitators from the Royal Tropical Institute (KIT) in the Netherlands, to introduce the various modules and provide technical guidance during the development of the research proposals.

The participants were divided into four groups. Before the workshop, it had been decided that it would be preferable to limit the area of the research to topics related to prevention of disabilities (POD) in leprosy. Due to the background and specific interest of some of the participants, at the beginning of the workshop integration of leprosy services into the general health services was added as a research topic for one of the groups. The remaining three groups chose topics related to POD in the phase before a patient is started on MDT, the phase during MDT and the phase after release from treatment (RFT). During the workshop, the groups developed a full research protocol each, including a detailed work plan and a budget. All protocols included qualitative as well as quantitative research methods. Qualitative methods to be used were focus group discussions, key informant interviews and semistructured questionnaires. The final proposals included one case-control study, one retrospective cohort study and two descriptive studies. The total budget was $\$ 15,000$ US for the four projects together, with costs of individual projects varying between $\$ 2000$ and $\$ 6000$ US.

The full titles were:

- Delays in presentation and start of treatment in leprosy patients: a case-control study of disabled and non-disabled patients in Hararge, West Shoa and ALERT hospital.

- Can disabilities be prevented during treatment? A study into the factors involved.

- Analysis of ulcer cases in ALERT and Wollo Leprosy Control Programme and prolonged hospital stay in the ALERT and Borumeda Hospitals.

- Knowledge and attitude of health professionals in relation to the involvement of General 
Health Service (GHS) staff in the management of leprosy in Southern Nations, Nationalities and Peoples Region (SNNPR), Ethiopia.

Once funding of the proposals had been secured, the participants carried out the respective researches, alongside their day to day professional commitments. The actual research phase started in October 1996, and lasted until the end of March 1997. All groups utilized a number of research assistants to carry out parts of the research, but it is important to realize that most of the work was done by the group members themselves.

During the last 2 weeks of April 1997, a second workshop aimed at data analysis and report writing was held at ALERT. All groups had implemented their research proposals. Some hypotheses had been confirmed, others rejected, but all groups had come up with unexpected, chance findings. Most of these were very valuable, and enriched the quality and depth of the research.

The research suggested that although ALERT has a well developed Management Information System, certain aspects of the leprosy control programme were not achieving the desired results.

In the case control study which addressed the delays between appearance of the first symptom and start of treatment in leprosy patients, cases were patients with disability grade 2 at the time of diagnosis, and controls were patients diagnosed during the same period, who had a disability grade 0 at diagnosis. The average delay before diagnosis in disabled new cases was over 2 years and seemed to be related to the level of stigma in the community, which remains distressingly high. One striking finding was that on average around $10 \%$ of the total delay from first symptom to start of treatment occurred in general clinics with qualified staff. Surprisingly, (disabled) cases incurred greater delay in being referred for leprosy treatment within the health services than controls. The full results of this study have been published elsewhere. ${ }^{3}$

In the retrospective cohort study which looked at the factors involved in the prevention of disabilities during treatment, it was found that out of 113 patients who technically fulfilled the criteria for steroid treatment, only 43 were recorded as having received steroids. This was much less than expected. From interviews one possible reason emerged, namely, that health workers in the ALERT leprosy control area are not supposed to start patients on steroids unless their supervisor approves. As the latter is not always present when the patient visits a clinic, this may lead to delays and some patients may even miss treatment for their new nerve function loss altogether.

Further, it was found that according to the patient cards, only $48 \%$ of MB patients who were treated with corticosteroids recovered satisfactorily, which is well below the $75 \%$ that are assumed to recover by ALERT staff. This may be partly a problem of definition and quantification of the degree of recovery, which needs further study.

Thus a number of important new insights were gained, some of which had important consequences for the programme at ALERT.

One group had had difficulties in carrying out all aspects of the research as planned. Lack of time, and the fact that the group members all worked at different localities were the major reasons for this.

The final output of the second workshop was four research reports, each with a full set of recommendations based on the findings of the research, and a plan of action as to how to implement these recommendations. The reports and the recommendations were presented to the relevant authorities during a meeting at ALERT at the end of the second workshop. 
It is expected that the next step will be that the plans of action will be implemented. Some of the recommendations will be implemented directly by the researchers themselves. Others will need to be discussed between the researchers and decision makers. In any case, the fact that the recommendations are based on findings from well designed and implemented research will increase the likelihood that they will be acceptable for these decision makers. It is further expected that findings will be presented at national symposia and seminars. Publication of at least one, but possibly two of the studies in international journals is anticipated. $^{3}$

\section{The future of HSR at ALERT}

At the end of the first, as well as the second workshop, written evaluations were held, whereby participants were asked to express their opinions on specific statements and score them from 1 (strongly disagree) to 5 (strongly agree). After both workshops, the participants turned out to be very satisfied with the process of the training. Although all agreed that they had benefitted from the course (mean score 4.6), a common complaint after the second workshop was that the time available for data analysis and report writing ( 2 weeks) had been too short. The fact that during the second workshop 2 days were 'lost' as a consequence of public holidays will no doubt have played a role in this matter.

The large majority of participants felt confident that 'there is a good chance the recommendations of our research will be implemented' (mean score 3.9), and they were 'looking forward to promote the utilization of our research' (mean score 4.3). Another positive effect of the training was that most participants were "confident to start other research projects' (mean score 4.4 ), and in fact $75 \%$ already had specific topics for study in mind, not only related to leprosy, but also to tuberculosis and other areas. While many staff at ALERT have had some involvement in research over the years, this was the first time for most participants to be involved in every stage of the research from beginning to end. This has given a much greater understanding of the purpose and process of research, and will incidentally help participants to evaluate other research more critically

A number of participants felt confident to be a facilitator in a possible future HSR training, which will enable ALERT to carry out similar HSR training courses with less (expensive) outside assistance.

It is recognized that it is too early to evaluate the actual impact of the training. Crucial questions which we hope to answer in a year's time are: have the recommendations that resulted from the studies been implemented? Have the problems that formed the justification for conducting the respective studies been solved or alleviated? Are participants applying some of the skills that they acquired during the training course in their day to day work?

\section{Contribution of HSR to leprosy control}

The experiences at ALERT suggest that HSR can make a useful contribution to leprosy control. Recently, similar workshops have been organized in other countries as well (e.g. India). The teaching material proved to be well suited for application to subjects related to leprosy, and no special adaptations were needed. 
Table 1. Leprosy research priorities for which HSR methodology is particularly suitable

- The development of more effective and efficient POD in leprosy control, primary health care and community based rehabilitation settings;

- The development of more effective self-care and footwear for patients with impairments;

- The development of methods to improve the implementation of MDT in inaccessible areas and to improve MDT completion rates;

- The development of strategies to implement MDT eff ectively through general health services and primary care in low endemic settings;

- The development of effective rehabilitation for leprosy patients within general rehabilitation programmes, including community based approaches.

Although operational research is not new in leprosy work, the methodology of HSR and certainly the systematic training of health workers in this methodology as well as the implementation of field research is a relatively new development. Training courses like the one described here not only serve to analyse and solve problems in leprosy programmes, but are a good investment in leprosy workers too, as they develop a broader view on the programmes they are working in.

In 1995, a short module on HSR was added to the well known, and widely used WHO training course 'Managing Programmes for Leprosy Control." ${ }^{2}$ As it is based on the same methodological concepts, it may be useful to use this module as a first step, whereby some participants who show keen interest in the topic are given the opportunity to undergo further HSR training along the lines of the courses described here.

Recently, the Medical Commission of the International Federation of Anti-Leprosy Associations, ILEP, has published a list of topics for future leprosy research. It argues that, as a result of the success of MDT programmes, the priorities of research in leprosy have changed. The Commission set new priorities for research in leprosy according to criteria such as feasibility, cost, time scale and proportion of patients benefiting, as well as the size of the benefit. ${ }^{4}$ It is exactly these criteria that can be used to characterize Health Systems Research. Some specific priority items mentioned in the Commission's report for which the HSR methodology seems particularly suitable are presented in Table 1.

\section{References}

${ }^{1}$ Varkevisser CM, Pathmanathan I, Brownlee A. Designing and conducting health systems research projects. IDRC and WHO, 1991, revised 1993.

2 Managing Programmes for Leprosy Control, Module 6. Exploring the need for research. WHO 1995.

${ }^{3}$ Bekri W, Gebre S, Mengiste A, Saunderson P, Zegwe S. Delay in presentation and start of treatment in leprosy patients: a case-control study of disabled and non-disabled patients in three different settings in Ethiopia. Int $J$ Leprosy, 1998; in press.

4 ILEP Medical Commission, Priorities for Leprosy Research 1996. ILEP Medical Bulletin Issue no. 10, October 1996. 